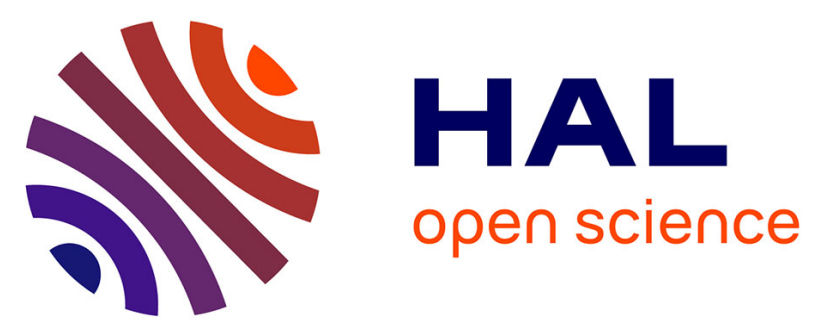

\title{
Challenges and opportunities in polysaccharides research and technology: The EPNOE views for the next decade in the areas of materials, food and health care
}

\author{
Zdenka Persin, Karin Stana-Kleinschek, Timothy J. Foster, Jan E.G. van \\ Dam, Carmen G. Boeriu, Patrick Navard
}

\section{To cite this version:}

Zdenka Persin, Karin Stana-Kleinschek, Timothy J. Foster, Jan E.G. van Dam, Carmen G. Boeriu, et al.. Challenges and opportunities in polysaccharides research and technology: The EPNOE views for the next decade in the areas of materials, food and health care. Carbohydrate Polymers, 2011, 84 (1), pp.22-32. 10.1016/j.carbpol.2010.11.044 . hal-00572426

HAL Id: hal-00572426

https://hal-mines-paristech.archives-ouvertes.fr/hal-00572426

Submitted on 9 Aug 2011

HAL is a multi-disciplinary open access archive for the deposit and dissemination of scientific research documents, whether they are published or not. The documents may come from teaching and research institutions in France or abroad, or from public or private research centers.
L'archive ouverte pluridisciplinaire HAL, est destinée au dépôt et à la diffusion de documents scientifiques de niveau recherche, publiés ou non, émanant des établissements d'enseignement et de recherche français ou étrangers, des laboratoires publics ou privés. 


\section{Challenges and opportunities in polysaccharides research and technology: The EPNOE views for the next decade in the areas of Materials-, Food- and Health Care.}

Z. PERSIN ${ }^{1}$, K. STANA-KLEINSCHEK ${ }^{1}$, T.J. FOSTER ${ }^{2}$, J.E.G. VAN DAM ${ }^{3}$, C.G. BOERIU $^{3}$, P. NAVARD ${ }^{4, *}$

1 Laboratory for Characterisation and Processing of Polymers - University of Maribor, Slovenia, Smetanova 17, SI-2000 Maribor, Slovenia

2 Division of Food Sciences, School of Biosciences, University of Nottingham, Sutton Bonington Campus, Loughborough, Leicestershire LE12 5RD, UK

3 Food and Biobased Products - Wageningen University and Research, Bornseweilanden 9, POB 17, 6700 AA Wageningen, The Netherlands

4 Mines ParisTech, CEMEF - Centre de Mise en Forme des Matériaux, CNRS UMR 7635, BP 207, 1 rue Claude Daunesse, 06904 Sophia Antipolis Cedex, France

This paper is a collective contribution of EPNOE members. European Polysaccharide Network of Excellence (www.epnoe.eu).

To contact EPNOE, use: contact@epnoe.eu

* Corresponding author. Tel.: +33 (0) 4939574 66; fax: +33 (0) 492389752.

E-mail address: patrick.navard@mines-paristech.fr (P. Navard). 


\begin{abstract}
The European Polysaccharide Network of Excellence (EPNOE) is a research and education network connecting 16 academic and research institutions and a large number of companies with its focus on polysaccharide expertise development and polysaccharide-related research for innovation in business and industry. EPNOE has two main missions in the field of polysaccharide applications in materials, food, and pharmacy/medicine, which are to organise education in polysaccharide science and to perform basic and applied research for the development of new products derived from polysaccharides. In 2009, the EPNOE network prepared a research road map vision to 2020 focussed on polysaccharide use in material structuring, food and health, taking both research and education into consideration. The research road map was prepared from various social, political, industrial and scientific inputs coming from within and outside EPNOE: (1) results of four brain-storming sessions by EPNOE scientists and students, (2) individual contributions of EPNOE scientists and (3) individual contributions of scientists outside EPNOE through an internet review. The result is described in this article.
\end{abstract}

\title{
Key-words
}

Polysaccharides, research, products, road map, EPNOE 


\section{Table of content}

1. Introduction

2. Fundamental basis of polysaccharide science

2.1 Identification of new polysaccharide sources

2.2 Structure, extraction, dis-assembly and isolation of single polysaccharides or polysaccharide-based blocs

2.3 Development of characterisation methods

2.4 Polysaccharide-polysaccharide and polysaccharide-non-carbohydrate interactions

2.5 In-vitro (enzymatic, chemical, mechanical/physical) and in-vivo (promoting natural sources to tailor polysaccharide functionality) modifications

3. Materials

3.1 Biobased materials.

3.2 Dis-assembly

3.3 Re-assembly

3.4 End of Life Cycle

3.5 Economical and Environmental Assessments

4. Food

4.1 Dis-assembly

4.2 Re-assembly

4.3 End of Life Cycle

5. Health Care

5.1 Dis-assembly

5.2 Re-assembly

5.3 End of Life Cycle

6. Conclusion

References 


\section{Introduction}

The fierce public debate that has been evoked since the UN conference on Climate Change in Copenhagen December 2009 is ongoing at all levels of politics, science and in the business world, with different voices and explanations of the consequences for the future of the planet and different views on the need for a transition towards a $\mathrm{CO}_{2}$ neutral biobased economy or bioeconomy. Independent of which scenario will be unfolding, increasing population and globalisation of economies will have the consequence of a need for enhanced supplies of biobased resources. To this end, carbohydrates are crucial since these are the sugar based building elements which all living things on this planet feeding on. Carbohydrates are the result of photosynthetic $\mathrm{CO}_{2}$ fixation in plants and the central exchange and communication system between organisms. In the global bioeconomy, carbohydrates, e.g. sugars and polysaccharides, are the central source of energy within which an economic value is intrinsically entrapped. Polymeric carbohydrates (or polysaccharides) such as cellulose and chitin are natural polymers found abundantly in Nature as structural building blocks. Other polysaccharides (starch, inulin) provide stored solar energy in the form of sugar for fuelling cells.

Academic, research and industrial EPNOE members are convinced that polysaccharides will be at the central point of the world of tomorrow for sustainable fuel, food, materials and medicine production. Such a vision, supported by most political, social and economical powers, is at the origin of the revival of research in this field in recent decades. With such efforts, there is no doubt that biobased polymers, among which polysaccharides represent the major share, will see a tremendous increase in academic interest and fundamental understanding that will boost the development of applications, not only limited to replace the present oil-based chemistry, but also opening new fields and markets. Polysaccharides and polysaccharide-based polymers offer credible answers to the challenges faced by the World in terms of global sustainability. It is thus the right time to think about where the focus of research on polysaccharides for materials, in food and providing health benefits, should be in the next decade. 
Established with the help of the European Commission (EC), the European Polysaccharide Network of Excellence (EPNOE) is a research and education network connecting $16 \mathrm{EU}$ academic and research institutions and more than 25 companies from all over the World focusing on polysaccharide and polysaccharide-related research and business. The 16 institutions described in [EPNOE] are composed of top-ranked universities and research centres, which have developed scientific expertise and state-of-the-art technologies in polysaccharide-related disciplines including chemistry, enzymology, biotechnology, chemical engineering, mechanics, materials science, microbiology, physics and life cycle assessment. In the field of materials, food, and health care the main missions of EPNOE are to organise knowledge transfer through education in polysaccharide science and to perform basic and applied research for the development of innovative products composed of and derived from polysaccharides.

In 2009, the EPNOE network prepared a road map on polysaccharide research and development needs for the next decade, for the EC, with a broad scope encompassing materials, food and health, and considering both research and educational aspects [www.epnoe.eu]. The distilled research road map described here was prepared considering various social, political, industrial and scientific inputs coming from inside and outside EPNOE: (1) results of four brain-storming sessions by EPNOE scientists and students, (2) individual contributions of EPNOE scientists and (3) individual contributions of scientists outside EPNOE through an internet review. The resulting document aims at guiding EPNOE members and all stakeholders involved in carbohydrate polymers (research institutes, young scientists, companies, national and international agencies) to identify new activity areas relevant for their purpose.

Three main drivers strongly push the use of polysaccharides. The first is the emergence of a bioeconomy that increases the contribution of biobased products. The second is the fact that polysaccharides, presently used in all sectors of human activities like materials science, nutrition, health care and energy, are polymers with exceptional properties, far from being fully recognized, opening routes for completely novel applications. The third is the ubiquitous presence and renewable character of polysaccharides, making them the primary $\mathrm{CO}_{2}$ neutral candidates for the global transformation to a more sustainable world. Proper utilisation of polysaccharide resources has the potential of increasing biodiversity, food safety and sustainability, decreasing of $\mathrm{CO}_{2}$ emissions and pollution, and is suitable for the production of 
fuel and materials and sustainable food production. Such new trends are not without questions and the motivations for the breadth of polysaccharides usage can be challenged:

- A severe shortage of oil threatening the production of oil-based polymers may not occur for a long time, in particular considering that polymer production uses less than $5 \%$ of the extracted oil.

- The environmental assessment of using polysaccharide materials (as well as a lot of biobased materials) does not always show a benefit over petrochemical products.

- Bad planning and environmental management of resources could lead not to an increase, but to a decrease of biodiversity.

- Innovations like e-inked paper or numerical books could lead to a strong decrease of paper production, decreasing demand of cellulose pulps and thus affecting by-product availability.

- Recycling of bio-mass based materials could be impractical to implement.

- A massive increase of polysaccharides used for energy and materials competes with food supplies in terms of land use and crop production.

Addressing these challenges and threats as well as elaborating the advantages of polysaccharide utilisation (e.g. decrease of $\mathrm{CO}_{2}$ emissions, potential for innovative high value and performance products, and better use of unused by-products and wastes) is behind EPNOE efforts to build this road map. Regarding $\mathrm{CO}_{2}$ emissions, the whole chain of life from resource production until end of life has to be taken into account. This issue is addressed in paragraph 3.4.

The EPNOE Road Map is built around two main focus areas. The first, called "Fundamental basis of polysaccharide science", constitutes the second chapter of this article where scientific challenges common to all application fields associated with major socio-economic and technological factors are reviewed: (i) identification of suitable sources, including underutilized crops and new productions, influence of climate challenge, possibilities of using genetic modifications, issues around food/non-food competition, biodiversity and sustainable uses of resources, (ii) knowledge of structure-function relations, extraction, dis-assembly and isolation of single polysaccharides or polysaccharide-based blocs, (iii) development of characterisation and quantification methods, (iv) advanced knowledge of polysaccharide- 
polysaccharide and polysaccharide-non-carbohydrate interactions and (v) in-vitro (enzymatic, physical/mechanical, chemical, physical, and combinations thereof) structural design of polysaccharides and polysaccharide derivatives and possibilities for in-vivo (engineering of natural sources to tailor bio-produced polysaccharides) modifications.

This fundamental introduction is followed by the second main focus of applied research with three identified "Application fields": Materials, Food and Health Care, constituting the third, fourth and fifth chapters. For each of the application fields, three levels of product cycle are considered, (i) extraction (dis-assembly), (ii) conversion (re-assembly) and (iii) consumption (end of life cycle). In addition, the Materials field has a section on economical and environmental assessments.

The use of polysaccharides in the energy sector as well as the resource management of polysaccharides, although important, is not addressed here.

The content of this article is schematically summarised in figure 1.

\section{Figure 1: Content of the EPNOE research road map}

\section{Fundamental basis of polysaccharide science}

Polysaccharides, in many forms, play a central role in all living organisms for supply and storage of energy and/or structural integrity and protection of cells. The science for use of polysaccharide-based substances is well evolved in manufacturing of health and cosmetic products, food and feed production, and for cellulose derived materials (wood products, paper and cellulose derivatives, or textiles). Advances in the use of polysaccharides are closely linked to the ability of the scientific community to unravel the complexity of polysaccharides in Nature. Translation of this knowledge to practical applications is required to model and shape the various biological, physical and chemical inter-relations taking place and to invent new and improved characterisation tools. Five main research areas have been identified, described below. 


\section{Figure 2: Schematic content of the "Fundamental basis of polysaccharide science" chapter.}

\subsection{Identification of new polysaccharide sources}

Considering the need for increased supplies of $\mathrm{CO}_{2}$ neutral resources and the vast amounts of agro-industrial, pharma, and forestry residues that are liberated in production supply chains, under-utilized biomass resources must be systematically identified and studied, examples being marine crops (algae, sea weeds) or less common land crops. Alternatively, the novel possibilities for crop breeding and functional genomics must be targeted at the improved production of novel polysaccharide structures. The identification of these alternative resource possibilities should reduce the constraints of food/non-food competition, help to solve biodiversity issues and produce a bank of data for the future development of products allowing sustainable uses of resources.

\subsection{Structure, extraction, dis-assembly and isolation of single polysaccharides or polysaccharide-based blocs}

Gaining control through genetic or agronomic manipulations of polysaccharide bioassemblies is achievable with the aim of understanding (a) how biosynthesis arranges the various components of the cell wall as supra-molecular polysaccharide assemblies or in carbohydrate storage, (b) the polysaccharide structural organization (tailoring) during biosynthesis and (c) polysaccharide construction/layering, temporary structures and biological scaffolds. A general understanding of the nature of supra-molecular polysaccharide assemblies is needed in order to optimise their dis-assembly, mainly the interaction of polysaccharides in-vivo with other organic components e.g. lignin, the solvent influence on polysaccharide interactions (e.g. water, salts, sugars) and the physics and chemistry of water and dissolved chemicals in their interactions with polysaccharides. This involves the development of new modelling and new numerical simulation tools of crystalline, semicrystalline and supra-molecular assemblies (helices, electrostatic interactions) and surface 
characterisation (hydrophilicity / hydrophobicity, charge / smoothness / pore-size characterisation) beyond the state-of-the-art. There is a need to improve the isolation of selected components with new "green" extraction methods reaching absolute (complete) extraction (retaining molecular structure) or to perform controlled partial extraction keeping some degree of supra-molecular organisation, and to understand the basic conditions for hydrogen and ionic bond breakage during extraction. Knowledge of the detailed description of the various interactions between molecules in these complex assemblies is missing and must be developed further.

\subsection{Development of characterisation methods}

Separation, purification, modification and application of polysaccharides require the use of advanced analytical tools to accurately describe the degree of modification e.g. degree of substitution, chain length distribution, including degree of branching, chain conformation, crystallinity and solvent interaction in structures in relation to physical behaviour. These techniques must be applied to polysaccharides in the fields of materials, food and healthrelated research. Of particular importance is the link between polysaccharide structure, both at a molecular and network level, in order to allow the study of accessibility, swelling and porosity. While some of these data are available for rather pure substances and some basic ordered states, there is an urgent demand to develop new analytical methods describing the intermediate states of polysaccharides (swollen state, gel, non crystalline areas), the various transition layers (like surfaces) and the interactions between polysaccharides or between polysaccharides and small molecules. To achieve this, new analytical methods (e.g. immunochemical assays, bio-recognition and enzyme assisted cleavage) must be developed and existing ones improved for advancing in the structural characterization of polysaccharides.

\subsection{Polysaccharide-polysaccharide and polysaccharide-non-carbohydrate interactions}

Polysaccharides exhibit many molecular interactions (1) with other polysaccharides leading to network structure formation, (2) with other polymers (e.g. proteins, lignins and phenolics, synthetic polymers) and (3) with smaller molecules (e.g. water, and other (ionic) solvents, leading to swelling, dissolution). Knowledge and understanding need to be expanded in order to boost development of new products: differentiation between hydrated / dry state / never- 
dried state / swollen state, identification of amorphous and swollen state and recrystallisation, structure of hydrogen-bond networks and their changes during swelling, drying and dissolution, hydrophilic/hydrophobic interactions and accessibility of polysaccharide surfaces for chemical interactions (with enzymes / proteins or chemicals and solvents) in amorphous regions and in the swollen state.

Interactions of polysaccharides with small molecules form the chemical basis for sorption of substances, chemical reactivity and dissolution of the polymers. Fundamental knowledge about sorption behaviour including hydrogen-bonding and hydrophobic interactions is required to design materials for food and non-food materials and for medical and cosmetic applications based on slow release conjugates, scaffolds for cell culture growing, bioactive compounds, (anti-allergenic and anti-bacterial, antibiotic/ anti-viral), immunogenic properties and physiological interactions.

\subsection{In-vitro (enzymatic, chemical, mechanical/physical) and in-vivo (promoting natural sources to tailor polysaccharide functionality) modifications}

$\underline{\text { In-vitro, }}$, the main scientific areas where there is a need for deeper knowledge are the development of new media for enhanced efficiency of enzymatic catalysed treatments under extreme conditions (like elevated temperatures), in organic solvents and ionic liquids, or supercritical fluids. The improvement of the enzyme assisted catalytic efficiency is of interest for polysaccharide modifying enzymes with hydrolytic, oxidative/reductive and transferase activity as well as the development of selective enzymatic cleavage and attachments of chains, side groups and branches. This opens the possibilities for performing (i) hybrid physical / (bio)chemical treatments combining high temperatures, high hydrostatic pressures, supercritical fluids, enzymes, ultrasound, microwave, high flow rates and (ii) highly reliable regio-selective chemical treatments.

In-vivo, the genetic control of polysaccharide formation is very well regulated. The mechanisms of genetic control at cell level are well understood, but still require the means and triggers for selective manipulation for enhanced or diverted biosynthesis production. Crop breeding and genetic modification research has investigated the effects on plant physiology and has been able to improve yields and crop qualities. The effects of these approaches on the formation of bio-assemblies require more focus. It is important to establish the sequence of events at the cellular level during bioformation of structures and the structure-property 
relationships when new polysaccharides with specific functions are to be produced (functional genomics). Genetically-modified and physiologically-improved crops, including cell culture production and agronomic testing of manipulated crops are needed for understanding the various bio-assemblies and structure-properties relationships, with the aim of creating new materials or improved productivity. The tools of molecular biology must be used to alter the structure of starch and other storage polysaccharides within the plant material. Ultimately, work aimed at introducing alien structures in biological organisms, for example the production of poly-lactic acid (PLA) in modified crops should be studied. Manipulation of biosynthetic production by substitution of genes encoding for polysaccharide production inside the biological system (e.g. production of chitin in plants) could be a valuable way to produce new structures or accumulation of easier extractable resources.

\section{Materials}

\subsection{Biobased materials.}

In the field of renewable energy, biobased energy is facing competition with solar and wind resources. But in the field of materials based on carbon petrochemistry, the only renewable alternative source is from biobased resources. In this way, bioplastics (like polylactic acid or polyhydroxyalkanoates) have been developed and brought onto the market, showing some potential as commodity plastics. Estimations on the size of present day non-food markets for renewable products is feasible, e.g. [Market studies. Li Shen et al. (2009)] for bioplastics. The overall market comprises many different sectors and covers a wide range of traditional products from paper and timber wood to textiles, cosmetics, medicines, aromas and specialty chemicals, as well as emerging innovations in the bioplastics production or materials design. Some products are made fully from renewable resources while others are only partially biobased. Technological innovation in the field of materials science should impart novel functional properties in $\mathrm{CO}_{2}$-neutral materials, and increase the performance of bioplastics, biofilms, protective coatings, structural performance materials and minimize the environmental impact of the production (search for non-polluting methods). This requires novel tools for manipulation of structures and smart design of processes. Much of the existing technologies for synthetic polymer processing and material testing apply for biopolymers, but 
there are significant differences with respect to the role of water and hydrogen bonding. The affinity of polysaccharides for water implies dimensional instability when uptake (swelling) and drying (shrinking) occurs. At different water contents polysaccharides can pass through the rubbery to glassy state, losing their flexibility and changing their mechanical properties to become brittle. Polysaccharides are also susceptible to decomposition at higher temperatures and thermoplastic behaviour is restricted because dehydration or degradation occurs. Another aspect is that hydrated polysaccharides are susceptible to microbial attack and biodegradation, which needs to be prevented for durable use of materials. The search for alternative methods and tools for polysaccharide processing, modification and conversion into technical applications and commercial interesting materials deserves a high priority from a scientific research point of view.

\section{Figure 3: Schematic content of the "Materials" chapter.}

\subsection{Dis-assembly}

Separation / fractionation: Nature provides polysaccharide materials embedded in a complex matrix of compounds, which define the function of each specific assembly like plant cell walls, seed grain hulls or exoskeletons of insects and molluscs. The future use of polysaccharide materials is directly coupled to the availability of suitable methods to collect, extract and purify the desired fraction of polysaccharides up to the quality defined by the processing route and application. The method of extraction determines the degree of preservation of the original structural arrangements. Disruption of the coherent supramolecular network may affect the original structural integrity. Breaking of bonds and hydrolysis of linkages is often necessary for removal of "impurities". Important routes to follow for achieving expansion of the existing technologies for solubilisation and separation are:

- sequential eco-efficient extraction processes without structural degradation or loss of properties (new enzymatic processes) to yield high amounts of "pure" polysaccharide components e.g. separation of hemicellulosic polysaccharide fractions like glucomannans, xylans from cell wall materials (cellulose, pectins and lignins); 
- fractionation by sequential removal of various compounds / components (e.g. biorefinery approach);

- fractionation of polysaccharides in well-defined forms using new environmentallyfriendly chemicals (e.g. fully recyclable ionic liquids);

- controlled enzymatic degradation (homodispersed fragments of cellulose, cleavage of selected side groups and branches);

- new modified enzymes to perform enzymatic processes in emulsions, (organic) solvent, with reactions at interfaces and for combination of different processes (high pressure and enzymes, high temperature and enzymes; enzymes and plasma treatments, etc.);

- genetic modification of raw materials to facilitate and improve subsequent fractionation (e.g. trees with low lignin for more efficient cellulose extraction);

- high energy processes e.g. dissolving and degrading cellulose in supercritical water prior to fermentation or high pressures;

- controlled separation of preserved cell wall structural complexes;

- new isolation and purification methods for commercially interesting polysaccharides such as chitins, hemicelluloses, pectins, starches, mannans etc.;

- new methods for in vitro enzymatic preparation of highly crystalline cellulose;

- production of tailor made glucans like large cyclodextrines;

- production of new high viscosity extracellular polysaccharides by fermentation.

Modifications: when the heterogenous natural structural organisations are dis-assembled, there are then many options to modify the polymers before using them into re-assembled structures. Besides the high number of excellent polysaccharide materials available directly from Nature, an endless number of polysaccharide-based materials can be generated by modification in homogenous and heterogeneous states. Semi-synthetic and man-made biopolymers can be produced by combination of various technologies. Biotechnological methods (e.g. genetic modification techniques) will enlarge the range of available materials even further. While chemical and biochemical modification (e.g. via derivatisation of the polysaccharides from solution state) allows modification of the polymer as a whole, heterogeneous derivatisation will focus more on topochemical access of reagents or enzymes 
and thus will only change the properties of the part of the polymer "accessible" to the chemicals dissolved or suspended in the liquid or gaseous surrounding.

Methods for improving chemical and enzymatic conversion of polysaccharide structures must be studied, like regioselective derivatisation, introduction of anchoring groups, grafting of polymers, formation of hydrophobic polymers, modification of swelling behaviour and solubility and introduction of special functions for stimuli responsive behaviour. Biotechnological methods include enzymatic introduction of functional groups, oxidation, derivatisation (esterification or attachment of polysaccharide polymers as side chains) and formation of tailored polysaccharide polymers. Controlled polymer chain degradation and chain formation, particularly employing enzymatic methods, support the production of well defined polysaccharide materials with high potential for medical applications e.g. scaffolds, tissue engineering, and materials with anti-thrombotic or antimicrobial activity. It is of interest to design specific chemical methods for modification of polysaccharides like starch to improve its use in non-food applications. Examples are controlled oxidation, specific homogenous group substitution and cross-linking of starch granules, introduction of novel functionalities (incl. regio-selectivity). For this polymer, it includes the use of new solvents (incl. ionic liquids). Improved characterisation methods for starch are required particularly for measuring the distributions of substituents, the amylopectin fine structure and the molecular size of starch polysaccharides.

\subsection{Re-assembly}

Innovation in new high performance materials based on conversion of polymeric carbohydrates is of great interest. Existing expertise in food processing or paper making where polysaccharide hydrocolloids form a major ingredient can be applied in materials science and vice versa. The demands on material properties and conversion may provide new ways of functional food preparation and health care products. Innovations for polysaccharide based products and materials involve the design of new chemical structures and new ways of processing.

New structures: Construction of bio-inspired structures or mimic of tissues as found in plants or animals with functional, structural or barrier properties is a great challenge for green materials development. For the construction of plant cell walls, polysaccharide layers are 
synthesized from monomeric sugars that are supplied in soluble form from inside the plasma membrane. During growth and formation of the cell walls in plant tissues, the different layers are subsequently formed. The primary cell wall is starting from a loose and open, flexible net of pectinous structures into which subsequent cellulose layers are included which are more rigid and give strength to the cell wall thickness may proceed until sufficient strength is achieved. In the final stage when the cells have attained the ultimate dimensions, lignification of the walls may occur to impart further strength and barrier properties. A similar sequence of events can be used to design the processing of polysaccharides to form materials such as stable foams, emulsions and layered structures with similar sequences of polymer deposition and similar complexities. To achieve this, there is the need of an important effort to understand and tailor structures at all dimensions from nano to macroscale. The control of chain associations, network formation of helices and their ordering in layered structures needs to be improved, and is an area which is under exploited. Alignment of ordered polysaccharide molecules determines much of the physical properties of the material. The exact processing conditions need to be established for blending or formation of hybrid concepts of polysaccharides with other polysaccharides / modified polysaccharides / non-polysaccharides polymers to make polysaccharide extrusion, molding, and coating possible. Controlled deposition of polysaccharides on a surface or growth of mineral crystals on a polysaccharide template can be used for mimic of biomineralization (calcite, silica etc.) and to construct biomimetic materials. There is a wide perspective for applying polysaccharides with novel biotechnological methods for constructing shapes and surfaces or coatings on different (synthetic) polymer surfaces in order to make them biocompatible or bioactive and to make superstructural assemblies of colloidal sols with inclusion of functional nanoparticles. The use of compatibilized polysaccharide materials as fillers or reinforcing filaments in polymers can be improved. Technological development of simple methods for the manipulation of polysaccharides for microspherization and nanospherization is of interest for encapsulation of chemicals or nanoparticles and construction of dendritic structures based on polysaccharides.

For all these materials, understanding of structural properties and molecular interactions in the medium used (solvent or gas - solid surface) as well as improving the method of purification and ways to have homogeneity of molecular weight and distribution of side chains are required. 
New processing technologies: new processing technologies must be developed. Some promising ones are:

- 3D shaping of low moisture polysaccharide materials from solutions.

- Structural design by bio-inspired polysaccharide engineering (genetic engineering, enzymatic modification), and layer by layer template and scaffolding processing.

- Re-assembling in large hierarchical structural elements (sheets, films, rods, spheres, cells) using polysaccharides as building blocks. Polysaccharide building blocks that are suitable for construction of materials requires the development of tools to handle the molecules, for examples in the attachment of smart tags for orientation and promotion of self organisation, or sensors to detect interactions (e.g. boronic acid) and vectors to guide and transfer the polysaccharide structures to the desired spot. Moreover, controlled fixation of the chains when properly positioned may be achieved by covalent bonding of reactive side groups and cross-linking agents or added resins.

- Cellulose thermoplasticity as a result of a combination of new stable plasticising agents and selected physical processing environments would offer tremendous opportunities to develop new products.

- Processing of large scale production of polysaccharide whiskers and nanofibrils.

- Controlled modification of polysaccharide surfaces by oxidation with tempo-catalysis or by selected enzyme treatments can be used for introduction of carbonyl groups, which are suitable for attachment of lipophile side groups or grafting of polymers and introduction of selected cross-links.

- Ways to include nanoparticles for the large scale production of nanocomposites solgel derived hybrids / nanotubes should lead to new materials with controled properties or novel functionalities such as mesoporous materials. The availability of these tools will open possibilities for conceptual design of hierarchically structured materials, including reconstruction of structural composites from polysaccharides and hybrid materials, in combination with other biomass polymers.

Computational models will be needed to support the process design for most of these processes. 
Products: intensification of research should be directed towards both bulk products and specialty products and smart materials. Bulk products include copolymers assembled from different polysaccharide units, novel polymers synthesised from polysaccharide building blocks and hybrids and composites built form polymer blends or coated structures as 3Dmaterials. Some examples of the range of specialty products include:

- high performance and functional fibres,

- fibre-composites for innovative textile and technical products, textiles and technical processes (e.g. flame retardant, hydrophobic, high strength, conductive, antibacterial);

- films with defined surface and permeability properties for packaging (e.g. permeability, antioxidant function), and high transparency polysaccharides films;

- multifunctional polysaccharide/cellulose derivatives for polysaccharide/cellulosebased materials or drugs;

- high sorbing structures;

- stimuli responsive materials (e.g. changing structure, form, thickness dependent on moisture, temperature, incorporated indicators);

- high performance materials containing polysaccharide rod-like nano particles;

- membranes with selective barrier function;

- carbonized polysaccharide products;

- molecular recognition systems;

- new thickeners for oil drilling,

- cellulose nanopaper structures of high toughness.

\subsection{End of Life Cycle}

Growing public awareness on climate change and the necessity for $\mathrm{CO}_{2}$ neutral production demands product information for making the most environmentally sustainable choice. The product selection has to be supported with detailed Life Cycle Assessment (LCA) of products from cradle to grave. The products derived through biotechnology are only more sustainable than competing alternatives when objective calculations yield lower values for the net energy, net emission, etc. during the full chain from creation to disposal. The enhanced ecological performance of renewable products (e.g. $\mathrm{CO}_{2}$ production, non-renewable energy, renewable 
energy, water use, land use, human toxicity) should, therefore, be demonstrated unambiguously by detailed analysis of the total life cycle of products.

Intrinsically polysaccharides are biocompatible and biodegradable and will not accumulate in the environment. However when polysaccharides are chemically modified or embedded and blended in synthetic matrices the disposal may cause problems. The target for new materials is to design the functional properties in such a way that after their service they can be re-used, recycled or disposed of without negative impact on the environment. Options for disposal at the end of the product life cycle require that the materials can be fully degraded by environmental influences (temperature, UV light, water and microbial attack). Most polysaccharides are known to degrade under conditions where microbial attack and fermentation can occur. Mineralisation of polysaccharide derivatives may proceed substantially slower by different composting routes. It is essential to study the recyclability and decomposition of polysaccharide-based materials, especially when they are parts of complex products made with high melting temperature oil-based polymers.

\subsection{Economical and Environmental Assessments}

The depletion of fossil resources, climate change and toxicity impacts are among the most important issues that are currently on the environmental sustainability agenda. One of the key strategies pursued to resolve these issues is the use of biomass for the production of heat and power, liquid biofuels and biobased materials. There are different views about which role biomass use as a whole will play in the medium to long term (e.g. as compared to other forms of renewable energy and nuclear power) and moreover, the importance of the various types of biomass usage. In the field of materials based on fossil carbon petrochemistry, the only alternative source is from renewable $\left(\mathrm{CO}_{2}\right.$ neutral $)$ biobased resources.

Environmental LCA is a tool to assess competing options in terms of their environmental performance. It can provide a decision basis not only for process selection but also for process improvement by providing insight into the impacts of each process step. Among the biobased materials, polysaccharides are among the key options for the future and they therefore serve as highly relevant cases for LCA studies, including the development and testing of novel methodologies. Some prominent methodological and empirical challenges related to LCAs of biobased materials which need to be better developed and studied are the assessment of: 
- land use efficiency,

- land use changes and its impacts on carbon emissions and biodiversity,

- soil carbon losses and other forms of soil degradation,

- water use,

- fertilizer use including phosphates and

- development of widely accepted single-score methods.

Furthermore, very important issues which go beyond the realm of LCA only are economic aspects, the competition of food versus non-food and the assessment of other social aspects like the social acceptance of land uses. In order to cover these aspects, suitable assessment methods for sustainability are urgently required. Certification and normalisation of the various impact factors is of high interest for policy and marketing. Therefore priority should be given to identification of well defined topics which can serve as case studies for the development of novel methodologies and for their application like polysaccharide-based materials versus their petrochemical counterparts; competing feedstock for cellulosics, e.g. comparison of wood versus cotton linters versus bamboo; environmental assessment of biobased packaging films accounting for the lower barrier properties as compared to the petrochemical counterparts; competing processing options for cellulosics, e.g. ionic liquids versus conventional processes and novel processing (synthesis, separation and purification) making use of new, highly efficient membranes.

\section{Food}

Polysaccharides have always played a central role in preparation of food products primarily as a carbohydrate source but not less importantly being used as additives to impart consistency, structure and texture as thickeners, gelling agents and emulsifiers (e.g. E400 alginic acid, E401 sodium alginate, E410 locust bean gum, E416, karaya gum, E465 methylethyl cellulose, E1404 oxidised starch, etc.). To an extent, a distinction can be made between an ingredient that can perform some of the functions of an additive (for example wheat flour used to thicken a wide range of food products) and poorly digestible cell wall polysaccharides (i.e. cellulose, hemicellulose and pectin) being the critical components controlling the texture and structure 
of fruits and vegetables as well as its nutritional value. The use of polysaccharides as dietary fibre and functional food ingredients is a major issue in food research and development.

The indigestible or poorly soluble polysaccharides that are contained in food products are referred to as dietary fibre. These are also called non-starch polysaccharides (NSP). Dietary fibres are as yet poorly defined and all cell wall polysaccharides may be classified as such. A wide variety of plant polysaccharides from many sources such as soluble $\beta$-glucans, arabinoxylans, fructans (inulin) and pectins have been investigated for their potential as dietary supplements or smart food additives. In recent years, the role of non-digestible polysaccharides in food has been recognized and they are now finding entrance to the market as low calorie functional food ingredients and prebiotics. Nutraceuticals or health promoting food additives are becoming more and more important in food industries and many new functional roles for polysaccharides and their derivatives in health food will be developed in the near future.

The water extractable $\beta$-glucans from cereal grains and especially barley (Hordeum vulgare) and oats (Avena sativa) are known as cereal gums. These linear glucans have interspersed $\beta(1 \rightarrow 3)$ and $\beta(1 \rightarrow 4)$ linkages in the chain. Similar glucans are found in cell wall tissues of many Gramineae such as ryegrass (Lolium multiflorum), wheat (Triticum avaesticum), rice (Oryza sativa), bamboo (Arundinaria sp) corn (Zea mays), etc.

The main research directions to be taken for polysaccharides in the area of food applications are given below, grouped into three paragraphs, dis-assembly, re-assembly and end of life cycle.

Figure 4: Schematic content of the "Food" and "Health Care" chapters.

\subsection{Dis-assembly}


Bio-assembly/isolation/extraction and characterisation: the majority of polysaccharide additives used in food production are fully natural materials. A consequence of this is strong natural variations between materials from different sources. This is due to both genetic and environmental factors, but also influenced by process related issues, for example during extraction and refining / purification. We see three major opportunities and challenges here:

- As previously alluded to, an understanding of the plant genome will allow the structure of the polysaccharides in the plant to be modified both by accelerated breeding programmes and through genetic engineering. What is important for foods is to include a food end-use dimension in such programmes. For example a functional genomics program that is focussed on the tailoring of the polysaccharide molecular size distribution or branching and substitution degrees may result in crops that yield higher quality and quantity of the valuable food ingredients. Consequently, producing new high yielding disease resistant wheat will have much less value if it does not have functionality required for use in human foods.

- Global warming and water shortage will require crops that can survive in marginal soils, higher temperatures and lower rainfall environments. It is known that starch structure will change under these environmental stress conditions resulting in different behaviour in food, but the reasons for this are not yet understood. Search for new species and varieties and adaptation of crops that can be productive in sub-optimal climatic conditions is important for future food supply security.

- Optimization of extraction processes for maximum functionality and minimisation of negative factors (degradation of quality, contamination, product loss) are required. Using the knowledge gained in materials research for developing new methods for polysaccharide extraction with preservation of structural integrity and novel solvents as are studied for cellulose, could be used to assist in the extraction of better quality food polysaccharides.

To meet these opportunities and challenges, the understanding of biosynthesis of polysaccharides and the genetic control mechanisms must be improved. It is also necessary to develop further tools for polysaccharide characterisation. Two examples are starch granule and carrageenan preparations. The starch granule is a complex bio-assembly. Some progress has been made in the development of new starches with different amylose-amylopectin ratios 
and amylopectin fine structures (e.g. waxy maize with extremely high amylopectin and Hylon VII with high amylose content), but we still are not confident about measuring the molecular size of the two main starch polysaccharides, partly because of difficulties in extraction. It has been recognised for some time that there are health negative aspects associated with the low molecular weight "tail" in carrageenan preparations. We currently do not have the analytical capabilities of determining, with the precision required, the amount of this low molecular weight material $<50 \mathrm{kD}$. If this can be achieved, then it will allow fractionation and extraction procedures and source selection to minimise the levels of this undesirable low molecular weight material.

In the agro-industrial production of food and beverages, large quantities of biomass are not used for value addition. Residues liberated in the production (stems, leaves, shells, skins, seeds, etc.) may be selected for extraction of lipids, fibres, polysaccharides (e.g. pectins, mannans, galactans, inulins, etc.), proteins and other products by biorefinery or controlled biocascading of extraction processes.

\subsection{Re-assembly}

Central to the use of polysaccharides is the structuring of food products for improved perception.

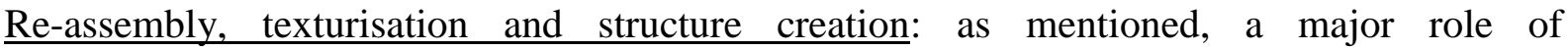
polysaccharide additives is to create structure in food products by acting as thickeners, gelling agents and emulsifiers. Gelation in particular can be regarded as a re-assembly process. The food industry is looking for new materials that can fulfil these functions without requiring additional chemical modification and preferable been considered as ingredients rather than additives. Examples are natural fibres, development of semi-refined materials and physical modification to alter functions. The use of natural fibres such as citrus fibres can be a cheap by-product from the fruit juice industry. There is scope for modifying such structures by combinations of physical, enzymatic and chemical treatments. However, to achieve this, a better understanding of the relationship between structure and function is required. The same considerations apply to the development of semi refined materials, building on the success of semi refined carrageenan. Physical modification can be interesting tools to alter the structure and function of an ingredient and in some cases replace chemically modified ingredients. An 
example is the relatively recent success in producing physically modified starches which have of the function of chemically crosslinked starches. Another example is extrusion processing of xanthan gum at low water contents to produce an ingredient that, unlike the unprocessed material, disperses readily and generates viscosity on heating.

Another important challenge is to produce low calorie (preferably low fat) products which match the organoleptic quality of current high calorie products. To achieve this, it is necessary to understand the relationship between product microstructure and perception. For example what microstructure is required for a high perceived creaminess? Therefore, further developments in the relationship between rheology and perception are required. The creation of the desired microstructure will be central to this, performed by developing particles fabricated primarily from polysaccharides which can trap water. Also important will be the development of polysaccharide nanoparticles. These "Pickering" particles, if fabricated with appropriate surfaces, have the potential of stabilising emulsions.

In order to create these new microstructures, an understanding of structure function relationships is critical. An important tool in progressing this understanding is the ability to selectively and specifically modify polysaccharide structures using both chemical and /or enzymatic methods. Of course the use of these tools must be accompanied by improved methods of determining polysaccharide fine structure.

Dried food products have the attraction of being shelf stable and light to transport. A fundamental problem is that the structure after drying and rehydration is different from the original structure. Learning about reversibility of water sorption and irreversible collapse of cellulose structures on rehydration will be utilised to develop dried food products of improved quality on rehydration.

Polysaccharide films will become increasingly important. Polysaccharide films are edible and as such can be used as barrier materials within food products. For example, reducing water migration between low water and high water environments in a pie can increase shelf life, also incorporation of polysaccharides into packaging film laminates can impart biodegradability and incorporation of chitosan to provide an antimicrobial function or sensors to measure oxygen levels can be used to develop active packaging films.

\subsection{End of Life Cycle}


The understanding of the relationship between diet and health will develop rapidly over the next ten years. This increased understanding will provide many opportunities for polysaccharides as food ingredients and also as medicines. The latter will be discussed in the next section on health. Largely because of their potential as thickening and gelling agents, polysaccharides can be used to control transit through the gastrointestinal tract (GI tract). For example, gastric emptying time can be reduced as a result of hydration/swelling of polysaccharide structures in the stomach or gelation in this acidic environment. This can increase satiety with the potential of reducing the level of obesity. Nutrients can be encapsulated in polysaccharides to provide controlled release. A potential negative effect is the undesirable consequence of fermentation of polysaccharides in the colon. To achieve these benefits without the disadvantages, a better understanding of the behaviour of polysaccharides in the GI tract is required. This will require development of physical/biochemical models of the digestive systems some of which are already available and the use of new techniques to image food in the GI tract (MRI imaging, confocal endoscopy) and animal studies. Additionally, an understanding of the interactions of food polysaccharides with mucilaginous secretions in the body will allow a full physico-chemical view of the structures involved in the digestive processes. Progress in this important area will come as a result of collaboration between polysaccharide scientists, nutritionists and medical scientists.

\section{Health Care}

Polysaccharides are a very diverse group of molecules, due not only to the different monosaccharide building blocks, but also the way constituent units are linked. Additional structural complexity is obtained by insertion of branches into the polysaccharide chains and side chain substitution with either organic or inorganic molecules. Unmodified polysaccharides are non-toxic, biocompatible, biodegradable, water soluble and have mostly high swelling ability and these are properties that make them extremely suited for biomedical, pharmaceutical and cosmetic applications. A wide range of polysaccharides have already found applications in human health and well being, namely chitosan, cellulose (both plant and bacterial) and cellulose derivatives, alginates, dextran, starch, hyaluronan, heparin, $\kappa-$ carrageenan, pectins and guar gums, in applications such as: 
- Drug delivery (starch and starch derivatives, cellulose and cellulose derivatives, chitosan, dextran, hyaluronan, pectin, carrageenan).

- Wound healing (chitosan, alginates, bacterial cellulose, hyaluronan)

- Tissue engineering scaffolds and implants (cellulose, hyaluronan, chitosan, alginates)

- Bioactive compounds as anti-microbial, anti-clotting of blood, drugs and vaccines (heparin, chitosan)

- Skin hydration, anti-aging agents, skin protection (antibacterial agents), (hyaluronan, chitosan, aloe)

- Vaccines (bacterial polysaccharides)

\subsection{Dis-assembly}

Disassembly, isolation/extraction and characterisation: polysaccharides used in health care and well being (cosmetic, hygiene) are natural materials derived from plants and animals or produced by microorganisms. This implies a natural variation that is further increased during the extraction and purification processes. In addition, most polysaccharide materials are naturally embedded into a complex matrix with other compounds, like proteins or lignin. The consequences of these intrinsic complexities have been discussed in detail in the two previous chapters and are valid for health applications also. However, for biomedical and pharmaceutical applications, there are much more strict criteria imposed regarding purity, homogeneity and consistent quality, including chemical structure and composition. Therefore, major challenges and opportunities are the development of improved processes for polysaccharide extraction, isolation and purification, with special attention to (i) the integration of new enzymatic, mild and non-destructive processes with mechanical processes and use of new solvents for the sequential extraction of polysaccharides to give high yields of highly pure polysaccharide components and (ii) the development of improved tools for the structural and molecular characterisation of polysaccharides, as well as tools for quantitative determination of trace impurities.

The specific focuses of polysaccharide for health-related applications are thus function and purity.

\subsection{Re-assembly}


The major roles of polysaccharides in biomedical and cosmetic applications are (i) to create structure i.e. in tissue engineering, scaffolds for cell growth or texturisers in body care products (in this respect, there is here a common focus together with the Materials and Food applications), (ii) to serve as carriers and protectants for drugs in the body from the administration point to the place in the body where the drug should be released to exert its activity and (iii) to act as bioactive materials i.e. anti-microbials or anti-clotting. The biomedical, pharmaceutical and cosmetic industry is looking for new materials that can fulfil these functions, being in the same time non-toxic, biocompatible and biodegradable.

Central to the use of polysaccharides is their property to form gels as well as more rigid structures upon dying, and this can be regarded as a reassembly process. Also, in most applications, reconstitution of complex spatial architectures by grafting the polysaccharides with other organic or inorganic polymers or blending to create new composite materials with new architecture is a major task. The main aspects that must be studied are:

- Development of new spatial architectures. For application of polysaccharides in tissue engineering, there is a need to improve their mechanical properties, as well as the thermal, chemical and biological stability. Ideal systems will be close to the nature-set parameters of sophistication, miniaturization, hierarchical organization, hybridisation, resistance and adaptability. These degradable and bio-resorbable polymeric structures must fulfil severe criteria related to biocompatibility and bio functionality. Polysaccharides can be tailored to bring specific behaviour in response to environmental stimuli, in which the design, chemistry, structure and function are intimately correlated. Important parameters are the morphology, the shape and size, the porosity, the size of the pores and the surface and surface functionality. There is a place for development of novel composite materials through blending or formation of hybrid concepts of polysaccharides with (1) organic and inorganic polymers and (2) with other polysaccharides or modified polysaccharides using various technologies. Another approach is coating of different synthetic polymer surfaces by polysaccharides in order to make them biocompatible or bioactive.

- Development of polysaccharide derivatives with multiple functionalities such as bioactive hydrogels. An example is native or modified chitosan as antimicrobial polymer in wound healing. Chitosan, however, has only a narrow spectrum of antimicrobial activity and have only limited applications in products due to its poor solubility at neutral and alkaline $\mathrm{pH}$. There is a need for developing new antimicrobial 
polymers derived from polysaccharides as antimicrobial non-leaching coatings for medical devices, antimicrobial thickeners in personal-care products and antimicrobial hydrogels for wound healing, in pharmaceutical and medical products. Important aspects in creating these bioactive hydrogels are the ability of selectively and specifically modifying the polysaccharide structure by inserting new side chains with the desired bioactive property using both chemical and enzymatic methods.

- Smarter polymers for target delivery of drugs. Due to their intrinsic properties, polysaccharides are extensively used for the delivery and controlled release of drugs. There is however the need of "smarter" drug delivery system. New materials like self assembly polymers, thermo sensitive and $\mathrm{pH}$ sensitive polymers exhibiting reversible sol-gel transitions or self-crosslinking must be developed. In order to prepare new materials able to target the drugs to a particular place in the body, through selective chemical and enzymatic modification of polysaccharides, there is a need:

- to change the hydrophilic-hydrophobic balance,

- to increase their hydrophobicity for targeting drug delivery to the colon, to add charges or reactive substituents for self-assembly or cross-linking and

- to crosslink the polysaccharides with other natural or synthetic polymers.

Structure-function relationships: In order to create these new nano- and microstructures, an understanding of the relationships between the structure of the new materials developed and their function and properties is critical. This is a request also needed in the Materials and Food areas, with a particular focus on the understanding of the interaction between polysaccharides and water and on the effects of hydrodynamic pressure. In progressing the understanding of structure-function relationships, several tools are needed, including the ability to selectively modify polysaccharide structures and to create new spatial architecture, the availability of methods of determining polysaccharide structure, and tools for determining the biological functions. This requires bringing together specialists from the fields of polysaccharide chemistry and physics, with material scientists and medical scientists.

New products: desired new products include high water content polysaccharide gels for wound healing, functionalised polysaccharide-based materials for target delivery of drugs, polysaccharide based thickeners and texturisers with antimicrobial properties, sensors, 
polysaccharide-based scaffolds for tissue engineering and the exploration of the use of new sources (microorganisms, algae, crop residues, and new crops, etc) to produce products.

\subsection{End of Life Cycle}

Most polysaccharides are biodegradable and prone to bacterial and enzymatic degradation, and this is an advantage for application in tissue engineering and drug delivery, but might be a problem for applications requiring a long life time, like polymeric antimicrobial agents in cosmetic formulations or packaging. The challenge is therefore to control the rate of degradation and the degradation time of polysaccharide based materials for each specific application. For example, cellulose is poorly biodegradable in the body and is not digestible, but it can be made hydrolysable by changing its high ordered structure.

The understanding of the relationship between the structure of the polysaccharide materials used in a specific application and its biodegradability as well as the ways/means of triggering biodegradability is essential. This will come from the use of physical and biochemical models of the in vivo degradation of biopolymers and requires the collaboration between polysaccharide scientists, biochemists and medical and cosmetic scientists. In health-related applications, the end of life cycle is strongly dependent on the function of the polysaccharide.

\section{Conclusion}

All recently published market studies [see ref Market studies] predict that the future of biomass-based polymers is bright, with polysaccharides and polysaccharide-derived polymers being in the forefront. This is in agreement with the huge potential of these polymers, as highlighted in this report. New insights in the structure and functions of polysaccharides in Nature open new ways for application developments though polysaccharide engineering. When considering the vast increase of research in this field in all parts of the World, we can expect to see the surge of a deeper understanding of the complexity of biomass, of its bioformation, of new ways to extract it while keeping the right level of molecular and structural integrity and more important of the detailed relations between structure and properties and functions. We believe that a new era for polysaccharide science is starting. 


\section{Acknowledgement:}

All EPNOE participants thank the European Commission for having given the opportunity to create this network (contract number NMP3-CT-2005-500375).

\section{References}

\section{$\underline{\text { Epnoe }}$}

EPNOE is organised through a legal, non profit organisation called EPNOE Association. At present, its members are 16 academic/research institutions and more than 25 companies. The 16 academic/research institutions are:

1. Armines CEMEF - Centre de Mise en Forme des Matériaux, BP 207, 1 rue Claude Daunesse, 06904 Sophia Antipolis Cedex, France, www.cemef.mines-paristech.fr

2. University of Natural Resources and Applied Life Sciences (BOKU), Department of Chemistry, Muthgasse 18, A 1190 Vienna, Austria, www.chemie.boku.ac.at

3. University of Jena, Centre of Excellence for Polysaccharide Research, Humboldtstrasse 10, D-07743 Jena, Germany, www.unijena.de/chemie/institute/oc/heinze

4. Fraunhofer-Institute for Applied Polymer Research, Geiselbergstraße 69, D-14476 Potsdam-Golm, Germany, www.iap.fraunhofer.de

5. VTT Technical Research Centre of Finland, VTT P.O. Box 1000, 02044 VTT, Finland, www.vtt.fi

6. vTI-Institute for Wood Technology and Wood Biology - University of Hamburg, Germany, Leuschnerstr. 91, 21031 Hamburg, Germany, www.vti.bund.de/en/institutes/htb

7. Åbo Akademi University ( ̊A), Porthansgatan 3, FI-20500 Turku/Åbo, Finland, www.abo.fi/fak/tkf

8. "Petru Poni" Institute of Macromolecular Chemistry, Aleea Grigore Ghica Voda, nr. 41A, 700487 Iasi, Romania, www.icmpp.ro

9. University of Maribor, Laboratory for Characterisation and Processing of Polymers, Smetanova ulica 17, 2000 Maribor, Slovenia, http://loppm.fs.uni-mb.si 
10. Agrotechnology \& Food - Wageningen University and Research, Bornsesteeg 59, POB 17, 6700 AA Wageningen, The Netherlands, http://www.biobasedproducts.wur.nl/UK/

11. Thüringisches Institut für Textil-und Kunstoff-Forschung e.V., Breitscheidstraße 97, 07407 Rudolstadt, Germany, www.titk.de

12. Institute of Biopolymers and Chemical Fibres with the incorporated Pulp and Paper Research Institute, ul. M. Sklodowskiej-Curie 19/27, 90-570 Lodz, Poland, http://www.ibwch.lodz.pl

13. University of Nottingham, Division of Food Sciences - School of Biosciences, Sutton Bonington Campus, Loughborough, Leicestershire LE12 5RD, UK, www.nottingham.ac.uk/biosciences/foodsci

14. University of Innsbruck, Research Institute of Textile Chemistry and Textile Physics, Hoechsterstrasse 73, A-6850 Dornbirn, Austria, http://www.uibk.ac.at/textilchemie/

15. Utrecht University, Department of Science, Technology and Society (STS) / Copernicus Institute, Heidelberglaan 2, NL-3584 CS Utrecht, Netherlands, www.copernicus.uu.nl

16. University of Graz, Institute of Chemistry, Colloid \& Polymers, Heinrichstraße 28, A-8010, Graz, Austria, http://chemie-graz.at/

\section{$\underline{\text { Market studies }}$}

Several market studies on biomass-based polymers and products appeared recently. We can cite:

1. Li Shen, Juliane Haufe, Martin K. Patel (2009), Product overview and market projection of emerging biobased plastics, Utrecht University. Commissioned by EPNOE and European Bioplastics, www.epnoe.eu

2. Ceresana research (2009): Market study bioplastics (UC-1105), Ceresana Research, Technologiezentrum, Blarerstrasse 56, 78462 Konstanz, Germany http://www.ceresana.com/en/market-studies/plastics/bioplastics/

3. Frost \& Sullivan's Technical Insights, Inc. Bioplastics (2005/09, Product code TI36481), 555 Eighth Avenue, Suite 902, Manhattan, NY 10018, http://www.ti.frost.com/prod/servlet/ti-home.pag 
4. Helmut Kaiser, Bioplastics Market Worldwide 2007-2025, hkc22@ bluewin.ch

5. Freedonia. World Bioplastics to 2013 - Demand and Sales Forecasts, Market Share, Market Size, Market Leaders. Study \#: 2548. Published: 11/2009. The Freedonia Group, Inc. 767 Beta Drive Cleveland,Ohio 44143, USA 


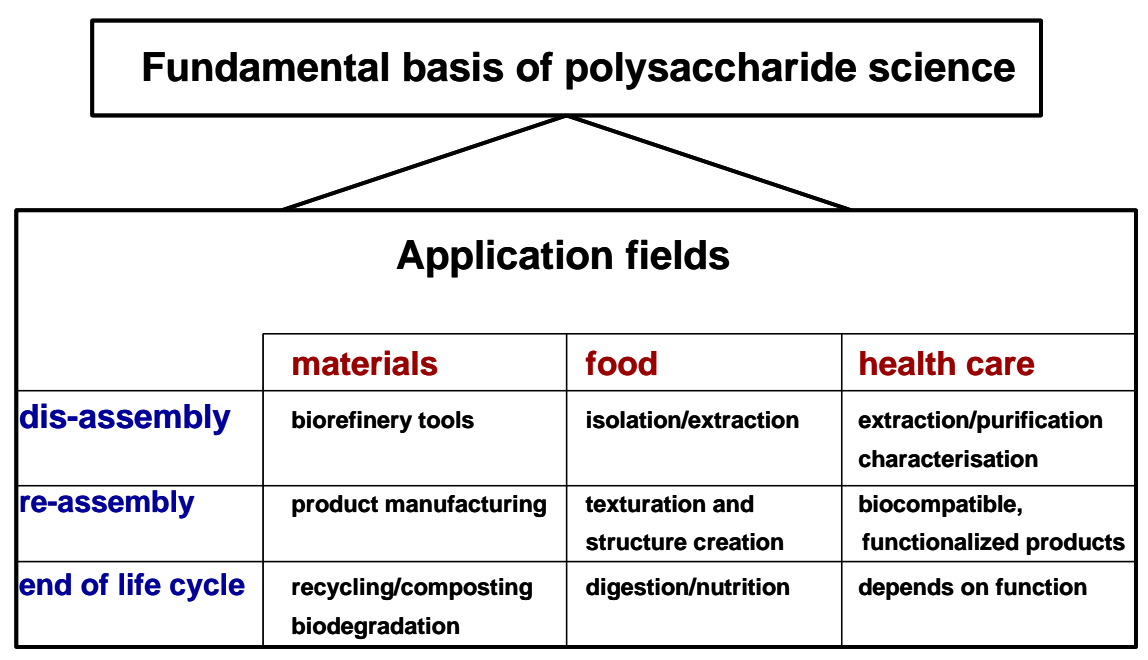

Figure 1: Content of the EPNOE research road map 


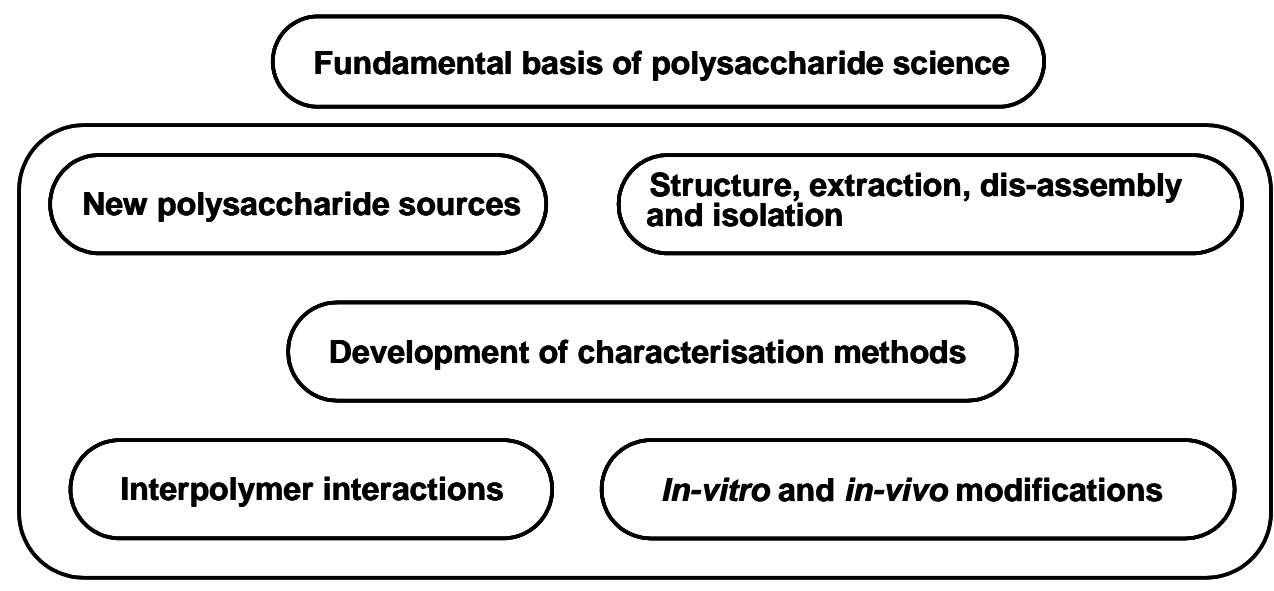

Figure 2: Schematic content of the "Fundamental basis of polysaccharide science" chapter. 


\section{Materials}

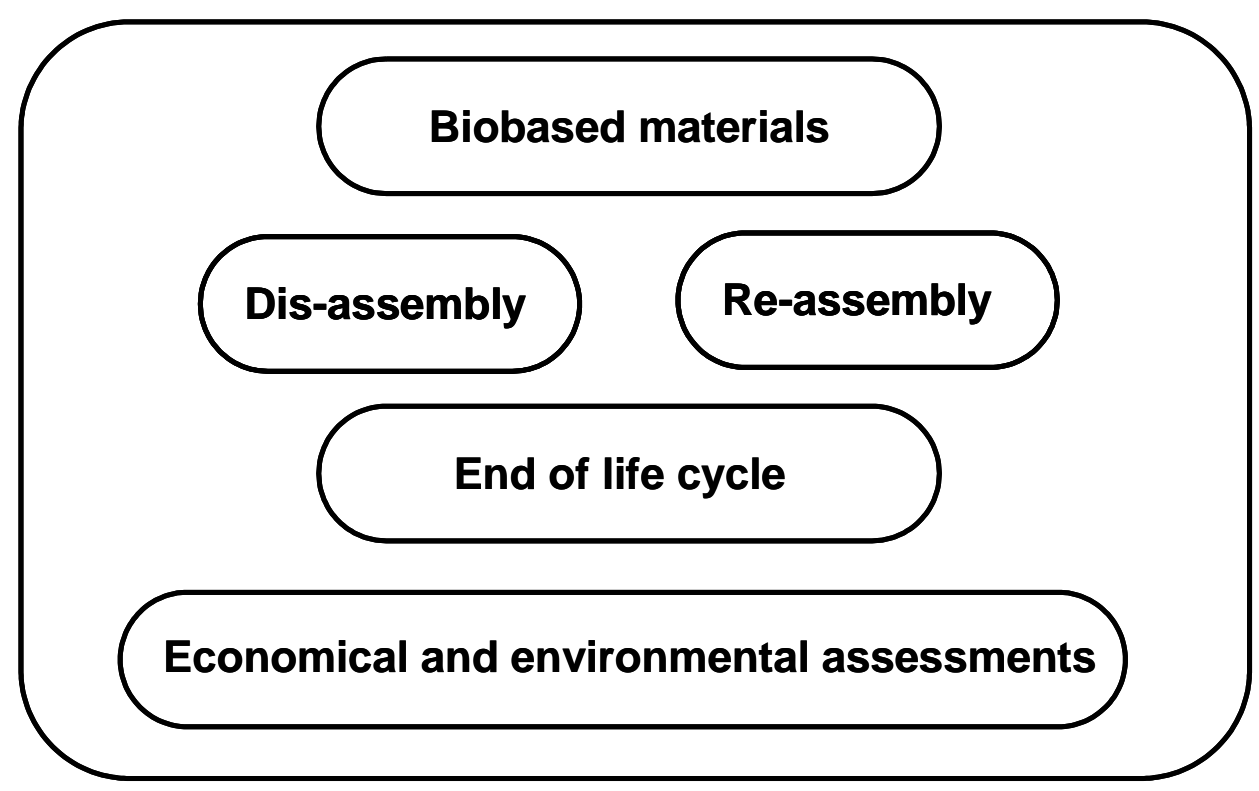

Figure 3: Schematic content of the "Materials" chapter. 


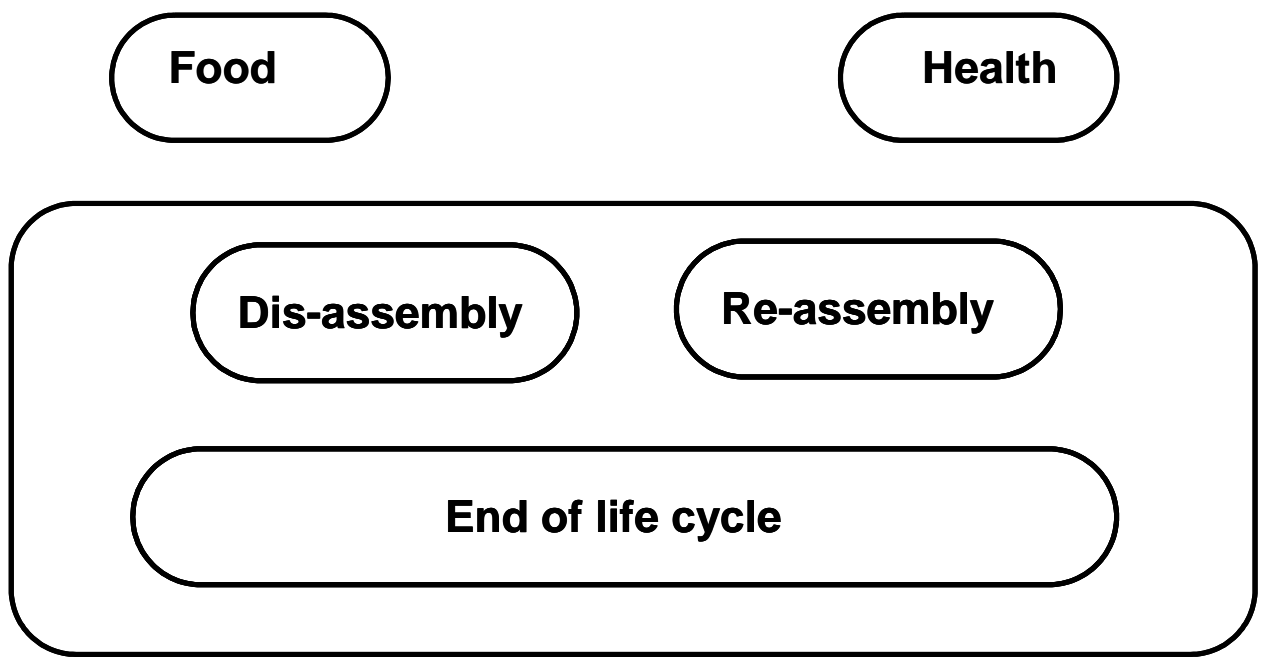

Figure 4: Schematic content of the "Food" and "Health Care" chapters. 